\title{
Community-Embedded Positive Mental Health Promotion Programs for the General Population: A Scoping Review Protocol
}

Lindsay K. Heyland ${ }^{1}$, RMT; Jodi E. Langley ${ }^{2}$, MSc; Sophie M. Keddy ${ }^{3}$; Alanna C. Kaser3; and Taylor G. Hill 4,5, MA

${ }^{1}$ Department of Psychology, Mount Saint Vincent University

2 Faculty of Health, Dalhousie University

3 Faculty of Science, Dalhousie University

4 Department of Psychology and Neuroscience, Dalhousie University

${ }^{5}$ Healthy Populations Institute, Dalhousie University

Taylor G. Hill (D) https://orcid.org/0000-0003-0048-7712

DOI: https://doi.org/10.15273/hpj.v1i2.11029

\begin{abstract}
Introduction: Positive mental health promotion (PMHP) is an emerging field within community mental health. Programming and policy efforts devoted to promoting mental health are developing. These efforts are varied in scope and nature, and there is little consensus on evidence-based best practices. Objective: To chart the body of literature on PMHP programming and to document the current PMHP in one Canadian province to provide insight into the types, scope, and nature of the programs currently and historically available to community residents in this province. Inclusion criteria: Peer-reviewed literature relevant to community mental health promotion, and grey literature that contains details of community-based programs accessible to the general population in that community. Methods: A preliminary search strategy in PubMed, EBSCO, and PsycInfo was developed with a librarian and a JBI-trained researcher. Primary studies published in English after 2000 evaluating or documenting PMHPs will be included. Grey literature from an environmental scan of existing local programs will be included. Data to be extracted includes study methodology and methods, program scope, content, materials, evaluation and outcomes.
\end{abstract}

Keywords: mental health; community; health promotion; well-being; program implementation 


\section{Community-Embedded Positive Mental Health Promotion Programs for the General Population: A Scoping Review Protocol}

\section{Introduction}

Positive mental health refers to a state of well-being that all individuals can achieve, regardless of whether they are experiencing mental illness, and is more than the absence of mental illness (Keyes, 2006; Orpana et al., 2016). The complete definition of mental health includes both salutogenic (positive states of mental functioning and capacity) and pathogenic (disease, disability, or premature death) perspectives (Keyes, 2013), and is characterized by high social, emotional and psychological functioning in everyday life (Keyes, 2002). As mental health is embedded within and influenced by a wider social, economic, and cultural ecology (Kobau et al., 2011), the determinants of positive mental health are inherently linked to the community in which one lives, works, and plays (Zubrick \& Kovess-Masfety, 2005). Positive mental health is an individual resource, contributing to the individual's quality of life, and can be promoted within the community (Herman \& Jané-Llopis, 2005). In the community, the act of promoting mental health seeks to strengthen individuals' psychosocial resources, enhance community institutions, and reduce structural barriers to positive mental health (Barry \& Friedli, 2010; Herman \& Jané-Llopis, 2005;).

Positive mental health promotion (PMHP) is an emerging field within community mental health. Community mental health is a system of care designed to reach specific populations of people in a way that is geographically accessible and locally relevant (i.e., program and services are tailored to the needs of the community; Caplan, 2013). It emphasizes the salutogenic perspective, suggesting that mental health is more than the absence of mental illness (Catalino \& Fredrickson, 2011), and encompasses our wellbeing and level of functioning in everyday life. Programming and policy efforts devoted to promoting mental health are emerging. These efforts are varied in scope and nature, and there is little consensus about which aspects of positive mental health to target, as well as unclear alignment with local health authority capacity. For example, community institutions such as schools, homes, work sites, places of worship, and health-care settings have been traditional targets for public health disease prevention and health promotion interventions, but are also promising settings for evidencebased PMHP interventions (Kobau et al., 2011).

Documenting PMHPs and related outcomes such as well-being can help in supporting and evaluating health promotion and public health wellness initiatives. A first step is charting the evidence base and identifying promising practices related to PMHPs that exist in communities. Reviews have been conducted with a focus on youth (Kuosmanen et al., 2019), and low-income countries (Barry et al., 2013), but, to date, the literature on community programs to promote positive mental health in non-clinical populations remains sparse. For example, a narrative review summarized PMHPs that were based in schools and designed for adolescents, finding that socio-emotional learning is a promising focus area for improving the mental health of young people (Kuosmanen et al., 2019). A systematic review focusing on youth in low- and middle-income countries found that school-based interventions tend to be successful in improving self-esteem and coping skills, although results vary by gender and age (Barry et al., 2013). A gap in current knowledge is the characteristics of PMHPs offered in community settings to the general public. To chart the literature on PMHPs, we will review the grey and peer-reviewed literature documenting the design and delivery of community-level PMHP programs. The grey literature will be documented through an environmental scan of existing programs available in the province (Nova Scotia). The peer-reviewed literature will be documented to chart the international body of literature describing PMHP programming.

A preliminary search of PROSPERO, MEDLINE, the Cochrane Database of Systematic Reviews, and JBI Evidence Synthesis was conducted, and no current or in-progress 
scoping reviews or systematic reviews on the topic were identified.

The objective of this review is to chart the body of literature on PMHP programming and to document the current PMHPs in one Canadian province to provide insight into the types, scope, and nature of the programs currently and historically available to community residents in this province. The context of mental and physical health in Nova Scotia includes low positive affect (e.g., happiness), and rates of chronic disease (e.g., chronic obstructive pulmonary disease) tend to be disproportionately high (Davidson et al., 2010). Conducting the environmental scan in one province is more feasible than conducting a national scan, and as all programs fall within one health authority (Nova Scotia Health), assessing the gap between current programs and international best practices will provide tangible recommendations aligned with the context of the province.

\section{Review Questions}

What is known about community-based PMHP programs for the general population?

Sub-question 1: What is the healthpromoting content of existing PMHPs? (e.g., teaching life skills, empowering individuals to become mental health literate)?

Sub-question 2: What methodologies, methods, measures, and tools have been used to study, assess, and implement these programs?

Sub-question 3: What is the evidence base for these programs?

\section{Inclusion criteria}

\section{Participants}

This review will consider studies that document mental health promotion programming within the community, available to the general public, without requiring a physician referral or a previous diagnosis.

\section{Concept}

PMHP programming within a community may include programs that teach basic life skills (e.g., emotion regulation, interpersonal communication), remove stigma related to mental health (e.g., improve mental health literacy), or guide individuals in therapeutic recreation or leisure activities such as personal projects, hobbies, or community interest group participation.

\section{Context}

This review is not limited to specific groups, places, or identities; rather, the review will consider all programs that are available to the general population. The environmental scan will be focused within a given geographical community, as explained previously.

\section{Types of Sources}

This scoping review will consider conceptual, quantitative, qualitative, and mixed methods study designs for inclusion. Systematic reviews and text and opinion papers will not considered for inclusion.

\section{Methods}

In consultation with a librarian and a Johanna Briggs Institute-trained researcher, a preliminary search strategy in PubMed, EBSCO, and PsycInfo was developed. The proposed scoping review will be conducted in accordance with the JBI methodology for scoping reviews (Peters et al., 2020). Scoping reviews address an exploratory research question by systematically searching, and selecting from, a wide range of literature to determine the breadth of evidence on a particular topic (Peters et al., 2020). Scoping reviews are designed to chart a body of literature with relevance to time, location, source, method, and origin (Levac et al., 2010). Our scoping review of peer-reviewed and grey literature will be complemented by an environmental scan of existing regional PMHP programs. Specifically, the scan of existing programs in Nova Scotia will provide baseline data on efforts by regional organizations and resources in the field of PMHP, identify gaps in evidence-based practices, summarize lessons learned, and identify challenges in the 
implementation of programs and opportunities for the delivery of future PMHP programming.

\section{Search Strategy}

The search strategy will aim to locate both published and unpublished primary studies. An initial limited search of MEDLINE (PubMed) and PsycInfo (EBSCO) was undertaken to identify articles on the topic. The text words contained in the titles and abstracts of relevant articles and the index terms used to describe the articles were used to develop a full search strategy for MEDLINE and PsycInfo (see Appendix A). The search strategy, including all identified keywords and index terms, will be adapted for each included information source. The reference lists of articles written by Margaret Barry (a pioneer of mental health promotion) will be screened for additional papers.

Articles published in English will be included, as our research team is limited to English speakers. Articles published from 2000 to the present will be included, as the evidencebased concept of PMHP emerged at this point in time. The databases to be searched include PsycInfo (EBSCO), MEDLINE (PubMed), Scopus, and CINAHL. Google Scholar will be used to identify grey and unpublished studies.

\section{Study/Source of Evidence Selection}

Following the search, all identified records will be collated and uploaded into Mendeley and duplicates removed. Following a pilot test, titles and abstracts will then be screened by two independent reviewers for assessment against the inclusion criteria for the review. Potentially relevant papers will be retrieved in full, and their citation details imported into Covidence. The full text of selected citations will be assessed in detail against the inclusion criteria by two independent reviewers. Reasons for exclusion of full-text papers that do not meet the inclusion criteria will be recorded and reported in the scoping review. Any disagreements that arise between the reviewers at each stage of the selection process will be resolved through discussion or with a third reviewer. The results of the search will be reported in full in the final scoping review and presented in a Preferred Reporting Items for Systematic Reviews and Meta-Analyses extension for Scoping Reviews (PRISMA-ScR) flow diagram (Tricco et al., 2018).

\section{Data Extraction}

Data will be extracted from papers included in the scoping review by two independent reviewers using a data extraction tool developed by the reviewers. The data extracted will include specific details about the mental health promoting content within the community program (e.g., the health-promoting skill or competency covered), the evidence base or theoretical background that the program is informed by, the methodologies, methods and measures that the program has been evaluated with, and the outcome of the program. A draft extraction tool is provided (see Appendix B). The draft data extraction tool will be modified and revised as necessary during the process of extracting data from each included paper. Modifications will be detailed in the full scoping review. Any disagreements that arise between the reviewers will be resolved through discussion or with a third reviewer. Authors of papers will be contacted to request missing or additional data, where required.

\section{Data Charting and Presentation}

The data extracted from relevant published and unpublished literature will be displayed to include the author and year of publication, type of source, intervention content, population(s), materials used, and documented outcomes. Data extracted from included papers will be presented in a tabular form, and the table will report key findings relevant to the review question(s). Data will be charted based on types of health-promoting content (e.g., life skills, emotion regulation). Results will be categorized under program type. A narrative summary will accompany the tabulated data and will describe how the results relate to the review objective and question. 


\section{Declarations}

Ethics Approval and Consent to Participate

This paper contains only publicly available information.

\section{Consent for Publication}

Not applicable.

\section{Availability of Data and Materials}

Data sharing is not applicable to this article as no data sets were generated or analyzed during the current study.

\section{Competing Interests}

The authors declare that they have no competing interests.

\section{Funding}

Not applicable.

\section{Acknowledgements}

Not applicable.

\section{References}

Barry, M. M., Clarke, A. M., Jenkins, R., \& Patel, V. (2013). A systematic review of the effectiveness of mental health promotion interventions for young people in low and middle income countries. BMC Public Health, 13, Article 835. https://doi.org/10.1186/14712458-13-835

Barry, M., \& Friedli, L. (2010). The influence of social, demographic and physical factors on positive mental health in children, adults and older people. In C. L. Cooper, J. Field, U. Goswami, R. Jenkins, \& B. J. Sahakian (Eds.), Mental capital and wellbeing (pp. 475-484). John Wiley \& Sons.

Caplan, G. (Ed.) (2013). An approach to community mental health (1st ed., Vol. 3). Routledge. https://doi.org/10.4324/97813150138 79

Catalino, L. I., \& Fredrickson, B. L. (2011). A Tuesday in the life of a flourisher: The role of positive emotional reactivity in optimal mental health. Emotion, 11(4), 938-950. https://doi.org/10.1037/a0024889 Davidson, K. W., Mostofsky, E., \& Whang, W. (2010). Don't worry, be happy: Positive affect and reduced 10-year incident coronary heart disease: The Canadian Nova Scotia Health Survey. European Heart Journal, 31(9), 1065-1070. https://doi.org/10.1093/eurheartj/ehp 603

Herman, H., \& Jané-Llopis, E. (2005). Mental health promotion in public health. Promotion \& Education, 12(S2), 42-47. https://doi.org/10.1177/10253823050 120020107

Keyes, C. L. M. (2002). The mental health continuum: From languishing to flourishing in life. Journal of Health and Social Behavior, 43(2), 207-222. https://doi.org/10.2307/3090197

Keyes, C. L. M. (2006). Mental health in adolescence: Is America's youth flourishing? American Journal of Orthopsychiatry, 76(3), 395-402. https://doi.org/10.1037/00029432.76.3.395

Keyes, C. L. M. (2007). Promoting and protecting mental health as flourishing: A complementary strategy for improving national mental health. American Psychologist, 62(2), 95-108. https://doi.org/10.1037/0003066x.62.2.95

Keyes, C. L. M. (Ed.) (2013). Mental well-being: International contributions to the study of positive mental health. Springer. https://doi.org/10.1007/978-94-0075195-8

Kobau, R., Seligman, M. E. P., Peterson, C., Diener, E., Zack, M. M., Chapman, D., \& Thompson, W. (2011). Mental health promotion in public health: Perspectives and strategies from positive psychology. American Journal of Public Health, 101(8), e1-e9. https://doi.org/10.2105/ajph.2010.300 083 


\begin{tabular}{|l|l|l}
\hline 1 & HEALTHY \\
POPULATIONS \\
JOURNAL
\end{tabular}

Kuosmanen, T., Clarke, A. M., \& Barry, M. M.

(2019). Promoting adolescents' mental

health and wellbeing: Evidence

synthesis. Journal of Public Mental

Health, 18(1), 73-83.

https://doi.org/10.1108/jpmh-07-

2018-0036

Levac, D., Colquhoun, H., \& O’Brien, K. K. (2010).

Scoping studies: Advancing the

methodology. Implementation Science, 5, Article 69.

https://doi.org/10.1186/1748-5908-5-

69

Orpana, H., Vachon, J., Dykxhoorn, J., McRae, L., \& Jayaraman, G. (2016). Monitoring positive mental health and its determinants in Canada: The development of the Positive Mental Health Surveillance Indicator Framework. Health Promotion and Chronic Disease Prevention in Canada, 36(1), 1-10. https://doi.org/10.24095/hpcdp.36.1.0 1

Peters, M. D. J., Godfrey, C., McInerney, P., Munn, Z., Tricco, A. C., \& Khalil, H. (2020) Chapter 11: Scoping Reviews. In E. Aromataris \& Z. Munn (Eds.), JBI Manual for Evidence Synthesis. JBI.

Tricco, A. C., Lillie, E., Zarin, W., O’Brien, K. K., Colquhoun, H., Levac, D., Moher, D., Peters, M. D. J., Horsley, T., Weeks, L., Hempel, S., Akl, E. A., Chang, C., McGowan, J., Stewart, L., Hartling, L., Aldcroft, A., Wilson, M. G., Garritty, C., ... Straus, S. E. (2018). PRISMA extension for scoping reviews (PRISMA-ScR): Checklist and explanation. Annals of Internal Medicine, 169(7), 467-473. https://doi.org/10.7326/m18-0850

Zubrick, S. R., \& Kovess-Masfety, V. (2005). Chapter 12: Indicators of mental health. In H. Herrman, S. Saxena, \& R. Moody (Eds.), Promoting mental health: Concepts, emerging evidence, practice (pp. 148-168). World Health Organisation. 